\title{
Radio Expansion Studies of Two Historical Supernova Remnants: SN 1006 and SN 1572
}

\author{
David Moffett, Caitlin Caldwell \\ Furman University, Greenville, SC 29613, USA \\ Estela Reynoso \\ Instituto de Astronomia y Fisica del Espacio, Buenos Aires, Argentina \\ John Hughes \\ Rutgers University, Piscataway, NJ 08854, USA
}

\begin{abstract}
We present results that trace the dynamics of two historical remnants, SN 1006 and SN 1572 (Tycho), for the past two decades. 21-cm radio images of each remnant were recorded during two previous epochs: 1983-1984 and 1991-1994. We obtained third-epoch images of both remnants from 2001 to 2003 . After a direct comparison of images from these three epochs, we found the mean radio expansion rate of Tycho now agrees with a previous X-ray expansion study, and for the first time, we have measured the azimuthal expansion rate over the entire rim of SN 1006.
\end{abstract}

\section{Introduction}

The expansion study of young supernova remnants can yield information on the interaction of high Mach-number interstellar shocks with the Galactic interstellar medium (ISM). As the supernova remnant (SNR) shock encounters and sweeps up material from the ISM, the shell expands non-linearly with time: $R \propto t^{-m}$ (where $m$ is the expansion factor). SN 1006 and Tycho have expansion factors of about 0.47-0.53 (Moffett, Goss \& Reynolds 1993; Reynoso et al. 1997), placing them both in a pre-adiabatic phase of SNR evolution.

\section{Procedure}

Third-epoch images of both SNRs were obtained with the Very Large Array. Additional observations of SN 1006 were conducted in 2003 with the Australia Telescope Compact Array. After completing standard amplitude and phase calibration, images of both remnants were CLEANed, convolved to a common beam width, and registered to a common pointing center using unresolved background radio sources. Radial amplitude slices were created from pie-shaped wedges at desired angles of azimuth and fitted for expansion using techniques discussed in Reynoso et al. (1997). 


\section{Results}

Our third-epoch observations of SN 1006 improved previous expansion measurements and have allowed us to measure the expansion rate over the entire rim of the SNR's shell (Figure 1). The expansion rates are typically $>0{ }^{\prime \prime} 40 \mathrm{yr}^{-1}$, and are consistent with optical expansion rates of filaments found along the northwest edge of the shell $\left(0^{\prime \prime} 30 \pm 00^{\prime} 04 \mathrm{yr}^{-1}\right.$; Long, Blair \& van den Bergh 1988). The northwest and southeast edges are expanding less rapidly than the rest of the shell.

Previous radio expansion measurements of Tycho yielded a mean expansion factor of $0.47 \pm 0.03$, lower than the expansion factor of $0.54 \pm 0.5$ derived from X-ray observations (Hughes 2000). This discrepancy prompted our third epoch observation of this remnant. Tracking the expansion of the radio shell through all three epochs, we find a mean expansion factor, $\mathrm{m}=0.53 \pm 0.03$, now in better agreement with previous X-ray measurements.

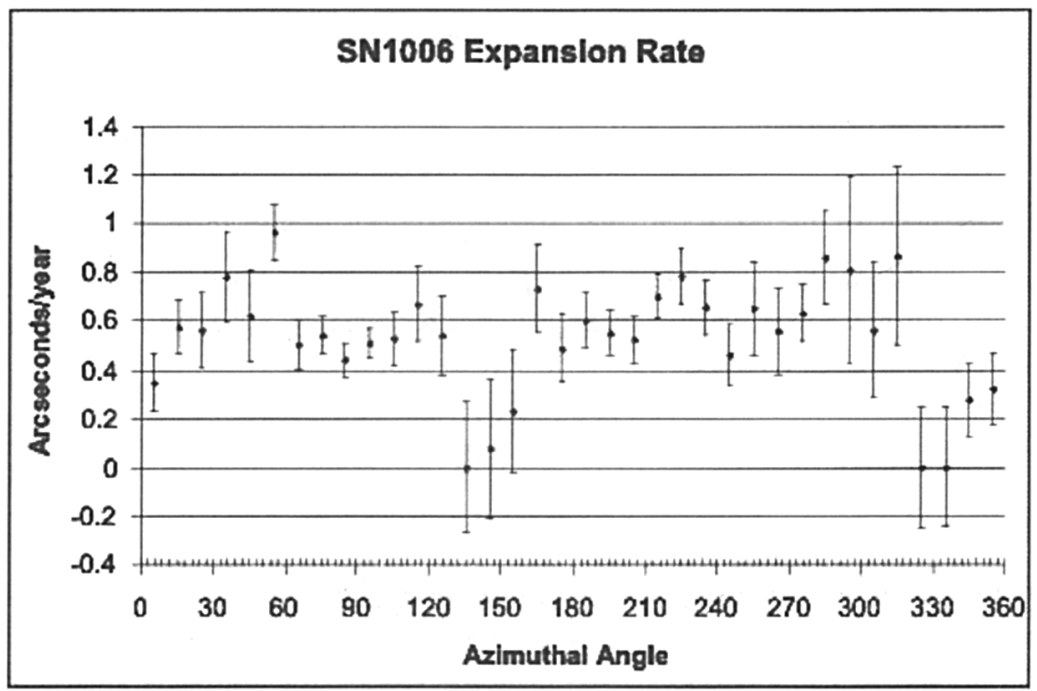

Figure 1. Expansion rate vs. azimuth angle for SN 1006.

Acknowledgments. D. Moffett thanks the American Astronomical Society for travel support to the 25th IAU General Assembly.

\section{References}

Hughes, J. P. 2000, ApJ, 545, L53

Long, K. S., Blair, W. P., \& van den Bergh, S. 1988, ApJ, 333, 749

Moffett, D. A., Goss, W. M., \& Reynolds, S. P. 1993, AJ, 106, 1566

Reynoso, E. M., Moffett, D. A., Goss, W. M., Dubner, G. M., Dickel, J. R., Reynolds, S. P., \& Giacani, E. B. 1997, ApJ, 491, 816 